ANNALES

POLONICI MATHEMATICI

$97.2(2010)$

\title{
Some characterizations of hyperbolic almost complex manifolds
}

\author{
by Fathi Haggui and Adel Khalfallah (Monastir)
}

\begin{abstract}
First, we give some characterizations of the Kobayashi hyperbolicity of almost complex manifolds. Next, we show that a compact almost complex manifold is hyperbolic if and only if it has the $\Delta^{*}$-extension property. Finally, we investigate extensionconvergence theorems for pseudoholomorphic maps with values in pseudoconvex domains.
\end{abstract}

Introduction. The concept of Kobayashi hyperbolicity has recently been extended to almost complex manifolds by several authors (see [7]-[9]). The main problem which interested those authors was to characterize the Kobayashi hyperbolicity of almost complex manifolds.

The main goal of this article is to investigate criterions for the Kobayashi hyperbolicity of almost complex manifolds and for extensions of pseudoholomorphic curves.

The paper is organized as follows: Section 1, which is essentially preliminary, contains the properties of almost complex manifolds used in the proofs of the main results. In Section 2, we give some characterizations of the Kobayashi hyperbolicity of almost complex manifolds. The first is furnished by a local estimate of the Kobayashi-Royden metric, which was proved by Royden [15] in the complex case. Then we define the Landau property and we prove that it is equivalent to hyperbolicity; this generalizes Hahn-Kim's [5] results.

In Section 3, we investigate the relationship between the $\Delta^{*}$-extension property and hyperbolicity for compact almost complex manifolds.

An almost complex manifold $(M, J)$ is said to have the $\Delta^{*}$-extension property if every pseudoholomorphic curve $f: \Delta^{*} \rightarrow(M, J)$ extends to a pseudoholomorphic curve $\tilde{f}: \Delta \rightarrow(M, J)$. It is known that each hyperbolic compact almost complex manifold has the $\Delta^{*}$-extension property (see [6]);

2010 Mathematics Subject Classification: 32Q45, 32Q60, 32Q65.

Key words and phrases: almost complex manifolds, Kobayashi hyperbolic manifolds, pseudoholomorphic curves. 
we prove a similar theorem without the compactness assumption and it is easy to show the converse.

Noguchi [13] proved a remarkable theorem about the extension and convergence of holomorphic maps. In [4], the authors proved several Noguchi type extension-convergence theorems in the almost complex case. We give some other variants of such theorems for almost complex maps; this generalizes the result of [17] proved in the complex case.

\section{Preliminaries}

\subsection{Almost complex manifolds}

1. Throughout this paper, by an almost complex manifold we mean a $\mathcal{C}^{\infty}$ smooth real manifold equipped with a $\mathcal{C}^{\infty}$ smooth almost complex structure $J$. Given two almost complex manifolds $(M, J)$ and $\left(M^{\prime}, J^{\prime}\right)$, a smooth map $f: M^{\prime} \rightarrow M$ is called $\left(J^{\prime}, J\right)$-holomorphic if its differential $d f: T M^{\prime} \rightarrow T M$ satisfies

$$
d f \circ J^{\prime}=J \circ d f .
$$

We denote by $\mathcal{O}_{\left(J^{\prime}, J\right)}\left(M^{\prime}, M\right)$ the set of $\left(J^{\prime}, J\right)$-holomorphic maps from $M^{\prime}$ to $M$.

2. For every $r>0$, we set $\Delta_{r}=\{z \in \mathbb{C}:|z|<r\}$, so $\Delta=\Delta_{1}$ is the unit disc in $\mathbb{C}$. If $\left(M^{\prime}, J^{\prime}\right)=\left(\Sigma, J_{0}\right)$ where $J_{0}$ denotes the standard complex structure on $\Sigma$ where $\Sigma$ is a Riemann surface, a $\left(J_{0}, J\right)$-holomorphic map is called a $J$-holomorphic curve or a pseudoholomorphic curve, and we denote by $\mathcal{O}_{J}(\Sigma, M)$ the set of $J$-holomorphic curves in $M$.

3. We say that an upper semicontinuous function $u$ on $(M, J)$ is plurisubharmonic if its composition with any $J$-holomorphic curve is subharmonic (see [3]). For a $\mathcal{C}^{2}$ function this is equivalent to the positive semidefiniteness of the Levi form:

$$
L_{u}^{J}(p, \xi) \geq 0 \quad \text { for any } p \in M \text { and } \xi \in T_{p}(M) .
$$

The value of the Levi form of $u$ at a point $p \in M$ and a vector $\xi \in T_{p}(M)$ is defined by

$$
L_{u}^{J}(p, \xi):=-d\left(J^{*} d u\right)(\xi, J \xi) .
$$

We remark that if $z: \Delta \rightarrow M$ is a $J$-holomorphic curve satisfying $z(0)=p$ and $d z(0)(\partial / \partial x)=\xi$ then $L_{u}^{J}(p, \xi)=\Delta(u \circ z)(0)$ (see [3]).

4. Throughout the paper, the following lemma due to Sikorav [16, Proposition 2.3.6, p. 171] will be useful.

Lemma 1.1. Let $D$ be a domain in $\mathbb{C}^{n}$. There is a positive constant $\delta_{0}$ such that for every almost complex structure $J$ in the neighborhood of $\bar{D}$ 
satisfying $\left\|J-J_{0}\right\|_{\mathcal{C}^{2}(\bar{D})} \leq \delta_{0}$, we have

$$
\|f\|_{\mathcal{C}^{1}\left(\Delta_{r}\right)} \leq c\|f\|_{\mathcal{C}^{0}(\Delta)}
$$

for every $f \in \mathcal{O}_{J}(\Delta, D)$ and every $0<r<1$, where $c$ is a positive constant depending only on $r$ and $\delta_{0}$.

1.2. Kobayashi hyperbolicity of almost complex manifolds. Let $(M, J)$ be an almost complex manifold. A Kobayashi chain joining two points $p, q$ in $M$ is a sequence of pseudoholomorphic curves $\left(f_{k}: \Delta \rightarrow\right.$ $(M, J))_{1 \leq k \leq m}$ and points $z_{k}, w_{k} \in \Delta$ such that $f_{1}\left(z_{1}\right)=p, f_{k}\left(w_{k}\right)=$ $f_{k+1}\left(z_{k+1}\right)$ and $f_{m}\left(w_{m}\right)=q$.

The Kobayashi pseudodistance of $(M, J)$ from $p$ to $q$ is defined by

$$
d_{M}^{J}(p, q)=\inf \sum_{k=1}^{m} d_{\Delta}\left(z_{k}, w_{k}\right)
$$

where the infimum is taken over all Kobayashi chains joining $p$ to $q$, and $d_{\Delta}$ denotes the Poincaré distance of $\Delta$.

Recall that for any two sufficiently close points $p$ and $q$ in $M$, there exists a $J$-holomorphic curve $u: \Delta \rightarrow M$ such that $u(0)=p$ and $u\left(z_{0}\right)=q$, where $z_{0} \in \Delta$.

For every $p \in M$, there is a neighborhood $\mathcal{V}$ of 0 in $T_{p} M$ such that for every $\xi \in \mathcal{V}$ there exist $r>0$ and a pseudoholomorphic curve $f: \Delta_{r} \rightarrow$ $(M, J)$ satisfying $f(0)=p$ and $f^{\prime}(0)=\xi$. This allows us to define the Kobayashi-Royden infinitesimal pseudometric $K_{M}^{J}$ :

$$
K_{M}^{J}(p, \xi)=\inf \left\{1 / r: f: \Delta_{r} \rightarrow M, J \text {-holomorphic; } f(0)=p, f^{\prime}(0)=\xi\right\} .
$$

The nonincreasing property of these pseudometrics can be stated as follows. Let $f:\left(M^{\prime}, J^{\prime}\right) \rightarrow(M, J)$ be a $\left(J^{\prime}, J\right)$-holomorphic map. Then

$$
f^{*} K_{M}^{J} \leq K_{M^{\prime}}^{J^{\prime}} \text { and } f^{*} d_{M}^{J} \leq d_{M^{\prime}}^{J^{\prime}}
$$

Kruglikov [8] extended Royden's results [15] and proved that $K_{M}^{J}$ is upper semicontinuous on the tangent bundle $T M$ of $M$ and that the integrated form of the Kobayashi-Royden metric $K_{M}^{J}$ coincides with the pseudodistance $d_{M}^{J}$ of Kobayashi. We say that $(M, J)$ is hyperbolic if $d_{M}^{J}$ is a distance.

Brody's reparametrization lemma [1] is valid for almost complex manifolds.

Lemma 1.2 (Brody's reparametrization lemma). Let $(M, J)$ be an almost complex manifold. Given a J-holomorphic map $f: \Delta_{r} \rightarrow M$ with $\left|f^{\prime}(0)\right| \geq c>0$, there exists a J-holomorphic map $\tilde{f}: \Delta_{r} \rightarrow M$ such that

$$
\sup _{z \in \Delta_{r}}\left|\tilde{f}^{\prime}(z)\right| \frac{r^{2}-|z|^{2}}{r^{2}}=\left|\tilde{f}^{\prime}(0)\right|=c .
$$




\section{Characterizations of hyperbolicity of almost complex mani-} folds. Let $(M, J)$ and $\left(M^{\prime}, J^{\prime}\right)$ be two almost complex manifolds. When $M$ is equipped with a length function $g, d_{g}$ will denote the induced distance, and for simplicity, we will write $|\xi|$ instead of $g(\xi)$ for $\xi \in T M$. As usual, $\Delta$ stands for the unit disk in the complex plane $\mathbb{C}$ and $\Delta^{*}=\Delta \backslash\{0\}$.

The theorem below gives a criterion for the hyperbolicity of an almost complex manifold. In the complex case, it is due to Royden [15].

TheOREM 2.1. Let $(M, J)$ be an almost complex manifold. Then the following statements are equivalent:

(i) $(M, J)$ is hyperbolic.

(ii) For every almost complex manifold $\left(M^{\prime}, J^{\prime}\right)$, the family $\mathcal{O}_{J^{\prime}, J}\left(M^{\prime}, M\right)$ is equicontinuous.

(iii) For any $p \in M$, there are a neighborhood $U$ of $p$ and a constant $c>0$ such that $K_{M}^{J}\left(\xi_{y}\right) \geq c\left|\xi_{y}\right|$ for all $\xi_{y} \in T_{y} M$ with $y \in U$.

Proof. (i) $\Rightarrow$ (ii). This is a direct consequence of the hyperbolicity of $(M, J)$ and the nonincreasing property of $\left(J^{\prime}, J\right)$-holomorphic maps with respect to Kobayashi pseudodistances.

(ii) $\Rightarrow$ (iii). Assume that condition (iii) is not satisfied. Then there exist $p \in M$, a sequence $\left(p_{n}\right)$ in $M$, and $\xi_{n} \in T_{p_{n}} M$ such that

$$
\lim p_{n}=p, \quad\left|\xi_{n}\right|=1, \quad \lim _{n \rightarrow \infty} K_{M}^{J}\left(p_{n}, \xi_{n}\right)=0 .
$$

Hence, there exists a sequence $\left(R_{n}\right)$ in $\mathbb{R}_{+}^{*}$ which tends to $+\infty$ and a sequence $\left(f_{n}: \Delta_{R_{n}} \rightarrow M\right)$ of $J$-holomorphic curves with $f_{n}(0)=p_{n}$ and $f_{n}^{\prime}(0)=\xi_{n}$.

Let $\left(g_{n}: \Delta \rightarrow M\right)$ be the sequence of $J$-holomorphic curves defined by $g_{n}(z)=f_{n}\left(R_{n} z\right)$. We have $g_{n}(0)=p_{n}$ and $\left|g_{n}^{\prime}(0)\right|=R_{n}$.

Let $W$ be a relatively compact neighborhood of $p$. By the hypothesis, there exists $r>0$ such that $g_{n}\left(\Delta_{r}\right) \subset W$ for $n$ sufficiently large. In view of Lemma 1.1, we have

$$
\left\|g_{n}\right\|_{\mathcal{C}^{1}\left(\Delta_{r / 2}\right)} \leq c_{r}\left\|g_{n}\right\|_{\mathcal{C}^{0}\left(\Delta_{r}\right)}
$$

which implies in particular that the sequence $\left(\left|g_{n}^{\prime}(0)\right|=R_{n}\right)$ is bounded. This is a contradiction.

(iii) $\Rightarrow$ (i). Let $p, p^{\prime} \in M$ with $p \neq p^{\prime}$ and $W$ be a relatively compact neighborhood of $p$ such that $p^{\prime} \notin \bar{W}$. Since $d_{M}^{J}$ is the integrated form of the Kobayashi-Royden metric, we have

$$
d_{M}^{J}\left(p, p^{\prime}\right) \geq c d_{g}(p, \partial W)>0 .
$$

An almost complex manifold $(M, J)$ is said to have the Landau property if for each $p \in M$ and each relatively compact neighborhood $W$ of $p$, there exists a positive constant $C$ such that

$$
\sup \left\{\left|f^{\prime}(0)\right|: f \in \mathcal{O}_{J}(\Delta, M) \text { with } f(0) \in W\right\} \leq C .
$$

As a consequence of condition (iii) in Theorem 2.1. we have the following 
Corollary 2.2. Let $(M, J)$ be an almost complex manifold. Then the following statements are equivalent:

(i) $(M, J)$ is hyperbolic.

(ii) $(M, J)$ has the Landau property.

This result is proved by Hahn-Kim [5] for complex manifolds. In particular, we obtain the following characterization of the hyperbolicity for compact almost complex manifolds.

Corollary 2.3. Let $(M, J)$ be a compact almost complex manifold. Then the following statements are equivalent:

(i) $(M, J)$ is hyperbolic.

(ii) $\sup \left\{\left|f^{\prime}(0)\right|: f \in \mathcal{O}_{J}(\Delta, M)\right\}<\infty$.

Using Brody's reparametrization lemma for almost complex manifolds, we obtain

Theorem 2.4. Let $(N, J)$ be an almost complex manifold and $M$ be a compact subset of $N$. Then only one of the following two statements holds:

(i) There exists an open neighborhood of $M$ in $N$ which is hyperbolic.

(ii) There exists a non-constant $J$-complex line in $M$.

By a non-constant $J$-complex line in $M$, we mean a non-constant entire $J$-holomorphic curve $u: \mathbb{C} \rightarrow N$ whose image is contained in $M$.

3. Characterizations of $\Delta^{*}$-extension for almost complex manifolds. Our main result in this section is that a compact almost complex manifold is hyperbolic if and only if it has the $\Delta^{*}$-extension property.

We recall that an almost complex manifold $(M, J)$ has the $\Delta^{*}$-extension property if every pseudoholomorphic curve $f: \Delta^{*} \rightarrow(M, J)$ extends to a pseudoholomorphic curve $\tilde{f}: \Delta \rightarrow(M, J)$.

EXAMPLE 3.1. Every hyperbolic compact almost complex manifold has the $\Delta^{*}$-extension property (see Remark 3.7).

First, we prove the following

Proposition 3.2. Let $(M, J)$ be an almost complex manifold. If $(M, J)$ has the $\Delta^{*}$-extension property, then $(M, J)$ has no $J$-complex line.

Proof. Assume that there exists a non-constant $J$-holomorphic curve $\sigma: \mathbb{C} \rightarrow(M, J)$, say $\sigma(1) \neq \sigma(-1)$. Consider a holomorphic map $g$ from $\Delta^{*}$ into $\mathbb{C}$ such that $g(1 / n)=(-1)^{n}$. Clearly, $\sigma \circ g$ does not extend.

Under the hypothesis that $(M, J)$ is hyperbolic, extensions of pseudoholomorphic curves defined on $\Delta^{*}$ can be characterized as follows: 
TheOREM 3.3. Let $(M, J)$ be a hyperbolic almost complex manifold and $u: \Delta^{*} \rightarrow(M, J)$ be a J-holomorphic curve. Then $u$ extends if and only if for some sequence $z_{k}$ in $\Delta^{*}$ with $z_{k} \rightarrow 0$, the sequence $u\left(z_{k}\right)$ stays in a compact set in $M$.

For the proof, we will need the following lemma:

Lemma 3.4. Let $(M, J)$ be a hyperbolic almost complex manifold, $u \in$ $\mathcal{O}_{J}\left(\Delta^{*}, M\right)$ and $p \in M$. Let $z_{k}$ be a sequence in $\Delta^{*}$ such that $z_{k} \rightarrow 0$ and $u\left(z_{k}\right) \rightarrow p$. If $\sigma_{k}=\left\{z \in \Delta^{*}:|z|=\left|z_{k}\right|\right\}$, then $u\left(\sigma_{k}\right) \rightarrow p$.

Proof. It is well known that the sequence of hyperbolic lengths $\ell\left(\sigma_{k}\right)$ satisfies $\ell\left(\sigma_{k}\right) \rightarrow 0$. Since, for $w_{k} \in \sigma_{k}$, we have $d_{M}^{J}\left(u\left(w_{k}\right), u\left(z_{k}\right)\right) \leq \ell\left(\sigma_{k}\right)$, it follows from the hyperbolicity of $(M, J)$ that $u\left(w_{k}\right) \rightarrow p$.

Proof of Theorem 3.3. We only have to prove the sufficiency of the condition. By the hypothesis, there is a sequence $z_{k}$ in $\Delta^{*}$ with $z_{k} \rightarrow 0$ such that $u\left(z_{k}\right)$ stays in a compact set in $M$. By considering a subsequence, we may assume that $u\left(z_{k}\right) \rightarrow p \in M$.

Assume that $u$ does not extend. Then there exist relatively compact local coordinate neighborhoods $W, U$ of $p$ such that $\bar{W} \subset U, W$ is diffeomorphic to the unit ball $B(p, 1)$ in some $\mathbb{C}^{n}$ centered at $p$ and there are sequences $z_{k}^{\prime}$ and $z_{k}^{\prime \prime}$ in $\Delta^{*}$ with $u\left(z_{k}^{\prime}\right) \in M \backslash U$ for each $k,\left|z_{k}^{\prime}\right| \rightarrow 0,\left|z_{k}^{\prime \prime}\right| \rightarrow 0$ and $\left|z_{k}^{\prime}\right|<\left|z_{k}^{\prime \prime}\right|<\left|z_{k}\right|, u\left(z_{k}^{\prime \prime}\right) \in \partial W$ for each $k$, and $u\left(z_{k}^{\prime \prime}\right) \rightarrow q \in \partial W$.

Let $G$ be a length function on $M$. Since $(M, J)$ is hyperbolic, there exists a positive constant $c$ such that

$$
K_{M}^{J} \geq c G \quad \text { on } U .
$$

By Lemma 3.4, we have $u\left(\sigma_{k}\right) \rightarrow p$, where $\sigma_{k}=\left\{z \in \Delta^{*}:|z|=\left|z_{k}\right|\right\}$.

Let $\mathcal{R}_{k}$ be the largest open annulus containing $\sigma_{k}$ with

$$
u\left(\mathcal{R}_{k}\right) \subset W .
$$

Since $u\left(z_{k}^{\prime \prime}\right) \rightarrow q \in \partial W$, there exist $a_{k} \geq\left|z_{k}^{\prime \prime}\right|$ and $b_{k}>\left|z_{k}\right|$ such that

$$
\mathcal{R}_{k}=\left\{z \in \mathbb{C}: a_{k}<|z|<b_{k}\right\} .
$$

We may assume that $a_{k}=\left|z_{k}^{\prime \prime}\right|$. Otherwise, there exists a sequence $w_{k}$ in $\Delta^{*}$ such that $\left|w_{k}\right|=a_{k}$ and $u\left(w_{k}\right) \rightarrow q^{\prime} \in \partial W$.

Let $\tilde{\mathcal{R}}_{k}=\left\{z \in \mathbb{C}:\left|z_{k}^{\prime \prime}\right|<|z|<\left|z_{k}\right|\right\}$ and $\rho_{k}=\left\{z \in \Delta^{*}:|z|=\left|z_{k}^{\prime \prime}\right|\right\}$. Again, by Lemma 3.4, we have $u\left(\rho_{k}\right) \rightarrow q$. Then for $k$ sufficiently large,

$$
u\left(\sigma_{k}\right) \subset B(p, 1 / 4), \quad u\left(\rho_{k}\right) \subset U \backslash \bar{B}(p, 3 / 4) .
$$

Therefore, there are points $c_{k} \in \tilde{\mathcal{R}}_{k}$ such that

$$
u\left(c_{k}\right) \in \partial B(p, 1 / 2) .
$$

Since all the curves $u\left(\tilde{\mathcal{R}}_{k}\right)$ are contained in $W$, by Gromov's monotonicity lemma [12] there exist positive constants $\varepsilon_{0}$ and $\alpha$ such that for $\varepsilon \in$ 
] $0, \inf \left(\varepsilon_{0}, 1 / 4\right)[$, we have

$$
\operatorname{Area}_{G}\left(u\left(\tilde{\mathcal{R}}_{k}\right)\right) \geq \operatorname{Area}_{G}\left(u\left(\tilde{\mathcal{R}}_{k}\right) \cap B\left(u\left(c_{k}\right), \varepsilon\right)\right) \geq \alpha \varepsilon^{2} .
$$

On the other hand, we denote by $\operatorname{Area}_{\Delta^{\star}}\left(\tilde{\mathcal{R}}_{k}\right)$ the area of $\tilde{\mathcal{R}}_{k}$ with respect to the Poincaré metric on $\Delta^{*}$. Then we have

$$
\operatorname{Area}_{\Delta^{\star}}\left(\tilde{\mathcal{R}}_{k}\right)=2 \pi\left(\frac{1}{\log \left(\left|z_{k}\right|\right)}-\frac{1}{\log \left(\left|z_{k}^{\prime \prime}\right|\right)}\right) \rightarrow 0 .
$$

It follows from (1) and (2) that

$$
\operatorname{Area}_{G}\left(u\left(\tilde{\mathcal{R}}_{k}\right)\right) \leq \frac{1}{c} \operatorname{Area}_{\Delta^{\star}}\left(\tilde{\mathcal{R}}_{k}\right) \rightarrow 0 .
$$

We get a contradiction.

REMARK 3.5. The monotonicity lemma of Gromov replaces Noguchi's arguments in [13] that exploited the Lelong numbers (see also [14] and [10, Theorem 3.6, p. 51]). We notice that the first author [2] proved that every closed positive current on an almost complex manifold possesses a Lelong number at any point.

As a consequence of Theorem 2.4, Theorem 3.3 and Proposition 3.2 , we easily deduce

Corollary 3.6. Let $(M, J)$ be a compact almost complex manifold. Then the following are equivalent:

(i) $(M, J)$ is hyperbolic.

(ii) $(M, J)$ has the $\Delta^{*}$-extension property.

REMARK 3.7. One can easily prove that each compact hyperbolic almost complex manifold has the $\Delta^{*}$-extension property.

Indeed, let $M$ be a compact hyperbolic almost complex manifold, $G$ be a length function on $M$ and $f: \Delta^{*} \rightarrow(M, J)$ be a $J$-holomorphic curve. By Theorem 2.1. there exists a positive constant $c$ such that $K_{M}^{J} \geq c G$, which implies that

$$
\left|f^{\prime}(z)\right|_{G} \leq \frac{1}{c} f^{*}\left(K_{M}^{J}\right)(z) \leq \frac{1}{c} K_{\Delta^{*}}(z) .
$$

Hence, the energy $E\left(f \mid \Delta_{r}^{*}\right)$ of $f \mid \Delta_{r}^{*}$ satisfies $E\left(f \mid \Delta_{r}^{*}\right) \leq\left(1 / 2 c^{2}\right) \int_{\Delta_{r}^{*}} K_{\Delta^{*}}^{2}(z)$ $<\infty$ for every $r \in] 0,1[$. Consequently, $f$ extends to a $J$-holomorphic curve $\tilde{f}: \Delta \rightarrow(M, J)$.

4. Extension-convergence theorems for pseudoholomorphic maps into pseudoconvex domains. The classical definition of pseudoconvexity can be extended to the almost complex case without changes.

Definition 4.1. A domain $\Omega$ in an almost complex manifold $(M, J)$ is pseudoconvex if for any compact subset $K$ in $\Omega$ its plurisubharmonically 
convex hull

$$
\hat{K}_{\Omega}=\left\{p \in \Omega: u(p) \leq \sup _{q \in K} u(q), u \in \operatorname{PSH}(\Omega)\right\}
$$

is compact.

Proposition 4.2. Let $(M, J)$ be a pseudoconvex almost complex manifold having the $\Delta^{*}$-extension property. Let $\left(f_{n}: \Delta^{*} \rightarrow(M, J)\right)_{n}$ be a sequence of J-holomorphic curves. If $\left(f_{n}\right)$ converges to a J-holomorphic curve $f: \Delta^{*} \rightarrow(M, J)$ uniformly on compact subsets of $\Delta^{*}$, then $\left(\tilde{f}_{n}\right)$ converges uniformly on compact subsets of $\Delta$ to $\tilde{f}$, where $\tilde{f}$ and $\tilde{f}_{n}$ are the extensions of $f$ and $f_{n}$ respectively.

Proof. By the hypothesis, it follows that $K=\bigcup_{n>1} f_{n}\left(\Delta_{s}\right)$ is relatively compact in $M$ for every $s \in] 0,1\left[\right.$. Since $M$ has the $\Delta^{*}$-extension property, it does not contain any $J$-complex line. Therefore, by Theorem 2.4, there is a hyperbolic neighborhood $W$ of $K$ in $M$. This implies that the family $\left(f_{n \mid \Delta_{s}}\right)$ is equicontinuous.

Since $\left(f_{n}\right)$ converges uniformly away from 0 , there exists a sequence $z_{n}$ in $\Delta^{*}$ converging to 0 such that $f_{n}\left(z_{n}\right)$ converges to $\tilde{f}(0)$. Hence, for every neighborhood $U$ of $\tilde{f}(0)$, there is $r \in] 0,1\left[\operatorname{such}\right.$ that $\tilde{f}_{n}\left(\Delta_{r}\right) \subset U$ for $n$ sufficiently large. This implies that the sequence $\left(\tilde{f}_{n}\right)$ converges to $\tilde{f}$ uniformly on some neighborhood of 0 .

We can generalize the $\Delta^{*}$-extension property to higher dimensional settings. We consider pseudoholomorphic maps defined on $X \backslash A$ with values in a pseudoconvex almost complex manifold having the $\Delta^{*}$-extension property, where $A$ is a thin subset in an almost complex manifold $X$.

Recall that a closed subset $A$ of $X$ is thin (cf. [6]) if there exists a local foliation $h$ of $X$ by pseudoholomorphic discs around $p$ for every $p \in A$, which satisfies the following conditions:

(A) There is a positive constant $r<1$ such that $A_{z^{\prime}}=\{w \in \Delta$ : $\left.h\left(z^{\prime}, w\right) \in A\right\}$ is a finite set contained in the disc $\Delta_{r}$ for every $z^{\prime} \in \Delta^{n-1}$.

(B) There exist sequences $\left(r_{j}\right)$ and $\left(s_{j}\right)$ of real numbers less than 1 such that $r_{j} \rightarrow 0$ and the cylinder $\left\{\left(z^{\prime}, w\right):|w|=r_{j},\left|z^{\prime}\right|<s_{j}\right\}$ intersects $h^{-1}(A)$ for no $j \in \mathbb{N}$.

For example, every smooth hypersurface in an almost complex manifold is thin. In particular, every smooth immersed pseudoholomorphic curve in an almost complex manifold of real dimension 4 is thin.

Finally, we mention that given a holomorphic map $u: X \backslash A \rightarrow M$, where $A$ is a thin subset, if $u$ extends continuously to $\tilde{u}: X \rightarrow M$, then $\tilde{u}$ is a pseudoholomorphic map (see [6]). 
TheOREM 4.3. Let $A$ be a thin subset of an almost complex manifold $X$ and let $M$ be a pseudoconvex almost complex manifold having the $\Delta^{*}$-extension property. Then every pseudoholomorphic map $u: X \backslash A \rightarrow M$ extends to a pseudoholomorphic map on $X$ into $M$.

Proof. For $p \in A$, choose a local foliation $h: \Delta^{d-1} \times \Delta \rightarrow X$ satisfying the conditions (A) and (B) in the definition of thin subsets. We denote by $u_{z^{\prime}}$ the map $u \circ h\left(z^{\prime}, \cdot\right)$ for every $z^{\prime} \in \Delta^{d-1}$. Since $u_{z^{\prime}}$ is a pseudoholomorphic curve defined on the unit disc $\Delta$ except at a finite number of points, it can be extended to a pseudoholomorphic curve defined on the entire disc $\Delta$ by the $\Delta^{*}$-extension property, for every $z^{\prime} \in \Delta^{d-1}$. We denote the extended map by $\widetilde{u}_{z^{\prime}}$. Let $z_{j}^{\prime}$ be a sequence in $\Delta^{d-1}$ which tends to 0 . As in the proof of Proposition 4.2, we may assume, choosing a subsequence if necessary, that the pseudoholomorphic mappings $\widetilde{u}_{z_{j}^{\prime}}$ converge to a pseudoholomorphic map $F: \Delta \rightarrow M$, uniformly on every compact subset of $\Delta$. Then it follows by the condition (A) that $F(w)=\lim _{j} \widetilde{u}_{z_{j}^{\prime}}(w)=u_{0}(w)$ whenever $|w|>r$. Therefore, $\widetilde{u}_{0}=F$ on $\Delta$ by the Unique Continuation Principle for pseudoholomorphic curves (cf. [11]) and $u_{z^{\prime}} \rightarrow u_{0}$ uniformly on every compact subset of $\Delta$ as $z^{\prime} \rightarrow 0$. This implies that $u \circ h$ is continuous in a neighborhood of $(0,0)$. Since $p$ is an arbitrary chosen point of $A, u$ can be extended to a continuous map defined on $X$.

Acknowledgments. The authors thank the referee for very useful comments that simplified some of the proofs and led to significant improvements in the paper.

\section{References}

[1] R. Brody, Compact manifolds and hyperbolicity, Trans. Amer. Math. 235 (1978), 213-219.

[2] F. Haggui, Sur l'existence du nombre de Lelong d'un courant positif fermé défini sur une variété presque complexe, C. R. Acad. Sci. Paris Sér. I 332 (2001), 299-304.

[3] - Fonctions PSH sur une variété presque complexe, ibid. 335 (2002), 509-514.

[4] F. Haggui and A. Khalfallah, Hyperbolic embeddedness and extension-convergence theorems of J-holomorphic curves, Math. Z. 262 (2009), 363-379.

[5] T. K. Hahn and T. K. Tim, Hyperbolicity of a complex manifold and other equivalent properties, Proc. Amer. Math. Soc. 91 (1984), 49-53.

[6] J. C. Joo, Generalized Big Picard Theorem for pseudo-holomorphic map, J. Math. Anal. Appl. 323 (2006), 1333-1347.

[7] S. Kobayashi, Almost complex manifolds and hyperbolicity, Results Math. 40 (2001), 246-256.

[8] B. Kruglikov, Existence of close pseudoholomorphic disks for almost complex manifolds and their application to the Kobayashi-Royden pseudonorm, Funct. Anal. Appl. 33 (1999), 38-48. 
[9] B. Kruglikov and M. Overholt, Pseudoholomorphic mappings and Kobayashi hyperbolicity, Differential Geom. Appl. 11 (1999), 265-277.

[10] S. Lang, Introduction to Complex Hyperbolic Spaces, Springer, 1987.

[11] D. McDuff and D. Salamon, J-Holomorphic Curves and Quantum Cohomology, Univ. Lecture Ser. 6, Amer. Math. Soc., Providence, RI, 1994.

[12] M.-P. Muller, Gromov's Schwarz lemma, in: Holomorphic Curves in Symplectic Geometry, M. Audin and J. Lafontaine (eds.), Birkhäuser, Basel, 1994, 217-231.

[13] J. Noguchi, Moduli spaces of holomorphic mappings into hyperbolically imbedded complex spaces and locally symmetric spaces, Invent. Math. 93 (1988), 15-34.

[14] J. Noguchi and T. Ochiai, Geometric Function Theory in Several Complex Variables, Transl. Math. Monogr. 80, Amer. Math. Soc., Providence, RI, 1990.

[15] H. Royden, Remarks on the Kobayashi metric, in: Lecture Note in Math. 185, Springer, 1971, 125-137.

[16] J.-C. Sikorav, Some properties of holomorphic curves in almost complex manifolds, in: Holomorphic Curves in Symplectic Geometry, M. Audin and J. Lafontaine (eds.), Birkhäuser, Basel, 1994, 165-189.

[17] D. D. Thai and P. N. Mai, Convergence and extension theorems in geometric function theory, Kodai Math. J. 26 (2003), 179-198.

Fathi Haggui, Adel Khalfallah

Institut préparatoire aux études d'ingénieur

rue Ibn-Eljazzar

5019 Monastir, Tunisie

E-mail: fathi.haggui@ipeim.rnu.tn

adel.khalfallah@ipeim.rnu.tn

Received 19.2.2009

and in final form 6.4.2009 